\title{
Design Guidelines for a Content-Based Image Retrieval Color-Selection Interface
}

\author{
Egon L. van den Broek, Peter M. F. Kisters, and Louis G. Vuurpijl \\ Nijmegen Institute for Cognition and Information \\ P.O. Box 9104, 6500 HE Nijmegen, The Netherlands \\ $+31(0) 243615476$ \\ e.vandenbroek@nici.kun.nl
}

\begin{abstract}
In Content-Based Image Retrieval (CBIR) two querymethods exist: query-by-example and query-by-memory. The user either selects an example image or selects image features retrieved from memory (such as color, texture, spatial attributes, and shape) to define his query. Hitherto, research on CBIR interfaces was absent. Hence, a usability evaluation of existing (CBIR) interfaces was done. Additionally, a study concerning human color cognition was conducted. Based on the resulting findings, a prototype color selection interface was developed. In a theoretical experiment, using Fitts' law, was proven that the prototype interface is highly efficient for CBIR purposes. This all resulted in a first set of design guidelines for CBIR color selection interfaces.
\end{abstract}

\section{Author keywords}

Content-Based Image Retrieval, CBIR, query-by-memory, color memory, color categories, color selection, Fitts' law, user-interface.

\section{ACM Classification Keywords}

H.5.2. [Information interfaces and presentation (e.g., HCI)]: User Interfaces - Graphical user interfaces (GUI); H.1.2 [Models and principles]: User/Machine Systems Human factors; I.4.7. [Computing Methodologies]: Image processing and Computer Vision - Feature Measurement.

Permission to make digital or hard copies of part or all of this work for personal or classroom use is granted without fee provided that copies are not made or distributed for profit or commercial advantage and that copies bear this notice and the full cit ation on the first page. To copy otherwise, to republish, to post on servers, or to redistribute to lists, requires prior specific permission and/or a fee.

Dutch Directions in HCI, June 10, 2004, Amsterdam

(C) 2004 ACM 1-58113-944-6...\$5.00

\section{INTRODUCTION}

The content of the World Wide Web consists to a great extent of images [8]. Given the exploding market on digital photo and video camera's, the fast growing amount of image content further increases the need for image retrieval systems. Unfortunately, most image retrieval systems are text-based and do not provide the means for searching on image content.

Content-Based Image Retrieval (CBIR) methods, however, are capable of searching in image collections, even when (adequate) textual annotations are missing. Instead of text they use either an example image as query (query-byexample) or a set of image features (query-by-memory). In the latter case, a complex interface has to be designed for facilitating the feature selection process, based on the users memory. This paper focuses on the design of a colorselection interface for query-by-memory in CBIR systems.

\section{LITERATURE OVERVIEW}

Hitherto, all research toward CBIR systems focused on the feature matching process underlying image retrieval. For query-by-example purposes, well performing feature extraction and pattern recognition technology is paramount. For query-by-memory retrieval, these techniques are equally important, but furthermore, a well-designed user interface is required. Nevertheless, no extensive review on user-interfaces of CBIR systems is present today. Existing CBIR reviews, such as that of Venters and Cooper [10] emphasize the various image retrieval techniques, but not their interfaces. Others such as Steiner [9] only briefly discuss the usability of 36 freely available web based colorselectors, in a non-CBIR setting.

Everly and Mason [6] did conduct a usability study specific on color-selection. They assessed speed, accuracy, and ease of use of four color-selection user-interfaces: Apple's Crayon (see Figure 1), HSV, RGB, and CMYK colorselectors. On all three criteria the Crayon color-selector, which uses a discrete presentation of 60 colors, outperformed the other three color-selectors. 


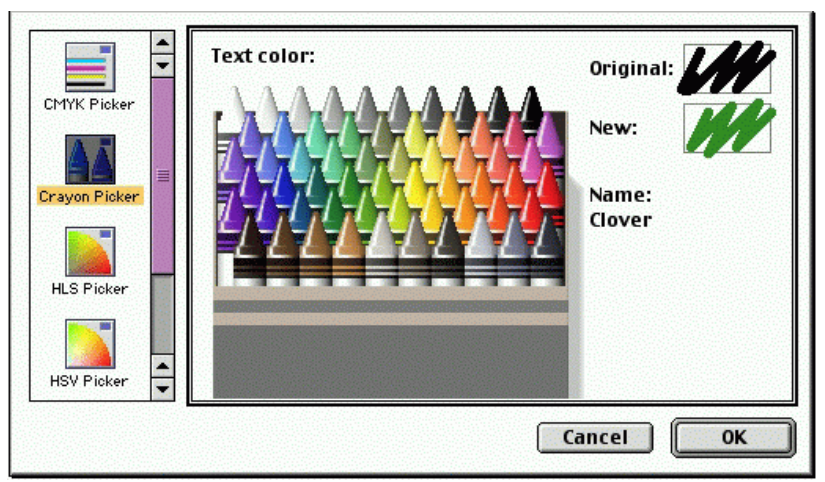

Figure 1. Apple Crayons color selector.

This can be explained by the origin of the HSV, RGB, and CMYK color-selectors: graphic design. Color-selectors in graphic design, available years before the first CBIR system was born, have other demands than color-selectors for CBIR. Subtle level crossings for example, do not have to be made in color-selection for CBIR, but are very custom in graphics design.

\section{EVALUATION OF 10 COLOR SELECTION INTERFACES}

In order to complete and sustain the latter study for CBIR user interfaces [6], 10 MSc.-students in Human-Computer Interaction were asked to evaluate the interfaces of 10 CBIR systems. They were all naive users in the field of CBIR. Concerning color selection, their findings can be summarized as a triplet of issues:

1. Most color selection interfaces require that the user is familiar with the presented color space. Imagine using three sliderbars, representing the Red, Green, and Blue axes of the RGB-model (see Java Sun RGB-sliderbars), for defining a color such as pink,

2. The students experienced the color selection interfaces as non-intuitive and often too "complex" (e.g., The interfaces provide multiple ways to define a color, which is confusing for the users.), and

3. The amount of variations of colors presented is eye-appealing, but judged as being not necessary.

Hence, in CBIR the need for differentiation between moreor-less similar colors was absent. The presentation of hundreds of colors was considered as overwhelming and with that as being inefficient. Nevertheless, current CBIR systems that allow the user to specify colors (see Table 1 and Figure 2), still exhibit such an interface.

The next two sections will underline that for basic color selection by memory, CBIR interface design can be optimized using (only) a limited set of colors.

\section{THE 11 COLOR CATEGORIES}

When defining a color by memory humans have to rely on color memory. It is well known that humans have a relatively poor color memory over the long term [4]. They tend to remember colors as members of categories. Most people distinguish 11-color categories: red, green, blue, yellow, brown, purple, pink, orange, black, white, and gray $[1,4]$.

There is a range of explanations for the existence of color categories; one of the strongest is the Sapir-Whorf view [11]. According to this view, linguistic categorization can influence non-linguistic perception and cognition. So, if colors appear more frequently than other colors, they are recognized more rapid than these.

Van den Broek et al. [2] confirmed the assumption that people quantize colors in 11 color categories. Next, they conducted two experiments that proved the difference between color categorization by the cognitive processes color discrimination and color memory. A color look-up table was derived from the experimental results. It was successfully used to design improved color matching techniques, yielding retrieval results that better match the query a user has in mind [3].

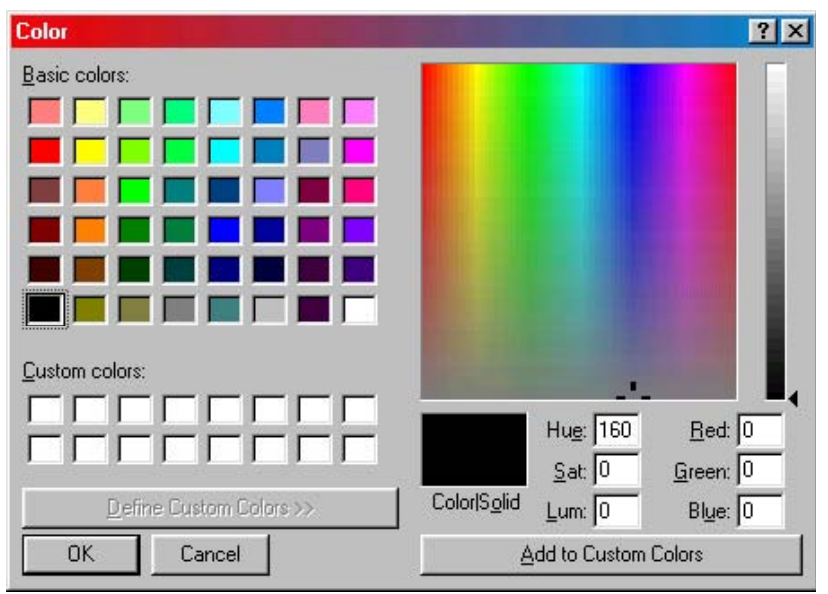

Figure 2. MS Paint and EditPlus color selector. The left part is the standard color selection interface. A design similar to that of the right part ("custom") of the interface is also used by MS PowerPoints "custom" color selector, by the QBIC, and by the SIMPLIcity color selection interface.

In addition, literature [4] states that color categories are relatively insensitive to various sources of variability, such as: illumination, memory, object identity, culture, and emotion.

Hence, the 11 color categories should be exploited for CBIR techniques and with that for the design of a queryby-memory CBIR color selector. The advantages of using them can be summarized as: 
1. They are robust to variability between people (i.e., all people are different and so is their color perception.) $[1,4]$,

2. They are robust to variability within people (i.e., and, for example, also changing perceptual abilities.) [1,4],

3. The small number of categories makes their use computationally very efficient [3], and

4. No color space is needed for displaying the color selection interface [6].

Below we will discuss another advantage of using this limited number of discrete color categories from a human motor point of view.

\section{A THEORETICAL EXPERIMENT: FITTS' LAW APPLIED ON COLOR-SELECTION USER INTERFACES Introduction}

An interface has a certain complexity from the perspective of human perceptual and motor skills. In 1954 Fitts [7] defined the Index of task Difficulty (ID), which best fitted his empirical results on human perceptual and motor skills.

The $I D$ is embedded in Fitts' law [7], which is expressed in:

$$
T=a+b \cdot I D,
$$

where $T$ is the selection time (i.e., the time needed to select the target). $a$ and $b$ are empirically determined constants that depend on the task to be fulfilled (i.e., the setting in which the target has to be selected), on motor, and on perceptual characteristics of the user. Fitts' $I D$ provides an indication for the motor workload of the color selection interface: the lower the $I D$, the lower the motor workload. An important goal as Dix et al. [5] already stated: "Speed and accuracy of movement are important considerations in the design of interactive systems."

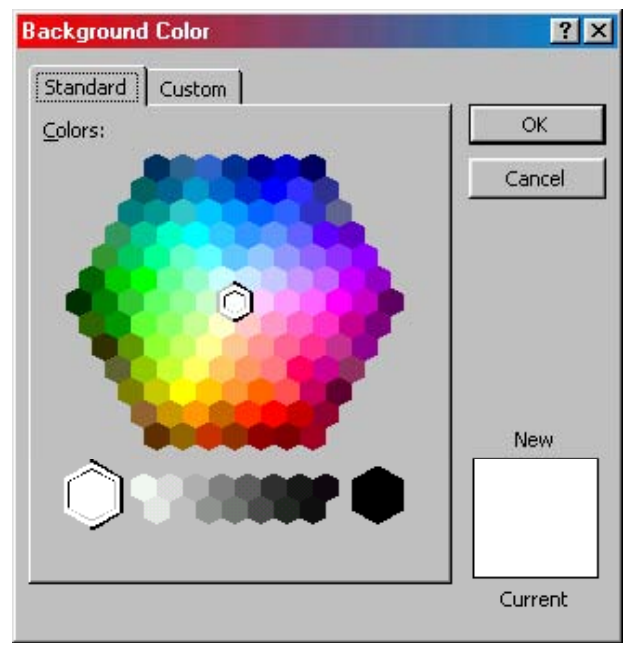

Figure 3. The MS PowerPoint standard color selector.

\section{Procedure}

The procedure discussed here can be applied on each selection system. First, for each cell (or color) $\varphi$ of the color selector under investigation, the Euclidean distance $\left(D_{\varphi}\right)$ was determined. This was defined as the distance from the center of the color-selection user-interface to the cell $\varphi$ :

$$
D_{\varphi}=\sqrt{x_{\varphi}^{2}+y_{\varphi}^{2}},
$$

where $x_{\varphi}$ and $y_{\varphi}$ are respectively the horizontal distance and the vertical distance between the center of the colorselection user-interface and cell $\varphi$.

Second, the minimum of width and height $\left(W_{\varphi}\right)$ of the cells was determined. Next, the $I D$ for each individual cell $\varphi$, was determined:

$$
I D_{\varphi}=\log _{2}\left(\frac{D_{\varphi}}{W_{\varphi}}\right)+1 .
$$

Third, the $I D_{\varphi}$ s of all cells $\varphi$ of the color-selector were summed and divided by the number of cells (or colors) $(\# \varphi)$ present in the color-selection interface:

$$
I D=\frac{\sum_{\varphi} I D_{\varphi}}{\# \varphi}
$$

This resulted in the $I D$ of the color-selection interface as a whole (see Table 1).

\section{Results}

The procedure discussed above was applied on nine colorselection user-interfaces. Four of them were part of an application (marked $\alpha$; see also Figures 1, 2, and 3), one of them was a stand-alone color chooser (marked $\beta$ ), and four of them were color-selectors of CBIR systems (marked $\varepsilon$ ). One of the latter was a prototype color-selector (see Figure 4 ). The results of the analysis were placed in Table 1. This table provides the name of the application, the average $I D$, the amount of cells (or \#colors) present, and the difference in $I D(\Delta)$, relative to the prototype color-selector.

\begin{tabular}{|l|r|r|c|}
\hline Name application & ID & \#colors & \multicolumn{1}{|c|}{$\boldsymbol{\Delta}$} \\
\hline MS PowerPoint standard $^{\alpha}$ & 2.32 & 144 & 1.97 \\
\hline MS PowerPoint custom $^{\alpha}$ & 3.27 & 810 & 2.79 \\
\hline MS Paint and EditPlus $^{\alpha}$ & 2.37 & 48 & 2.03 \\
\hline Apple's Crayon picker $^{\alpha}$ & 2.08 & 60 & 1.77 \\
\hline Java Sun Swatches $^{\beta}$ & 3.32 & 310 & 2.83 \\
\hline QBIC $^{\varepsilon}$ & 4.76 & 16384 & 4.05 \\
\hline SIMPLIcity $^{\varepsilon}$ & 4.76 & 32768 & 4.05 \\
\hline VisualSEEk $^{\varepsilon}$ & 1.88 & 56 & 1.60 \\
\hline Prototype color-selector $^{\varepsilon}$ & 1.17 & 12 & \\
\hline
\end{tabular}

Table 1. The color-selectors studied, with their Index of task Difficulty (ID), their amount of colors (\#colors), and the difference with the prototype color-selector $(\Delta)$. 


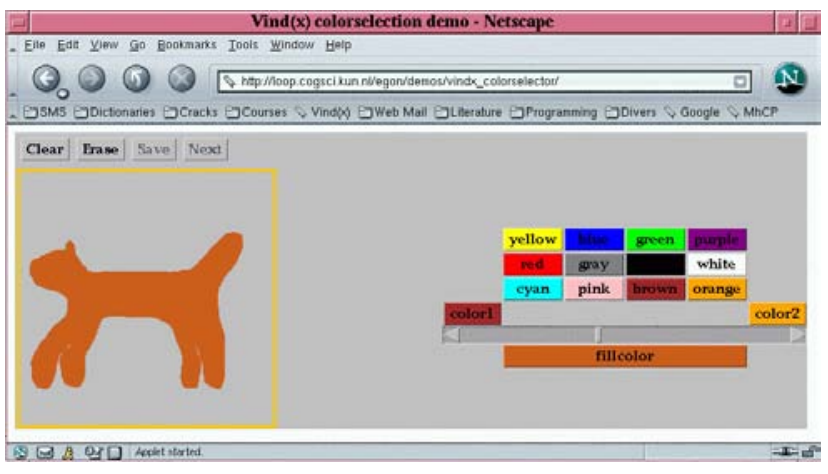

Figure 4. The prototype color selector, combined with a sketchpad for shape based image retrieval. It is based on human color memory the uses 11 color categories and cyan (the most used other color).

\section{Conclusions}

As can be observed in Table 1 the IDs of the analyzed color selectors differ enormously. QBIC and SIMPLIcity, with their eye-appealing 2-dimensional representation of a color space, have a high $I D$. Where simple color-selection userinterfaces, such as the VisualSEEk color-selector and Apples Crayon color-selector have a much lower $I D$.

The color-selector on the left of MS Paint and EditPlus (see Figure 2) seems to be an exception on this rule. This is due to the relatively small size of the cells and the wide space between them. This increased the relative distance that had to be traveled. So, a limited amount of colors is the most efficient from human motor point of view.

\section{DISCUSSION}

This paper uses three approaches to assess color-selection for query-by-memory in a CBIR setting. First, a usability evaluation of existing (CBIR) interfaces was done. Second, a study concerning human color cognition was conducted. This revealed that human color memory uses the 11 color categories to memorize colors. Based on the resulting findings, a prototype color selection interface was developed. Third, in a theoretical experiment, Fitts' law proved that the prototype interface did have the lowest motor workload and, therefore, is highly efficient (i.e., a very limited selection time).

Hence, for the design of a query-by-memory CBIR color selector one always has to take into account that:

1. Human color memory is poor and that it stores color in 11categories.

2. The more colors are present, the harder the selection is: both from perceptual and motor point of view.

3. Color-selectors in graphic design and in CBIR systems cannot be interchanged.
This paper discusses a prominent component of CBIR userinterfaces: the query-by-memory color selector. A first set of design guidelines is provided for query-by-memory CBIR color-selection interfaces. Based on these, a prototype interface was developed that is more efficient and has a high usability compared to other systems.

\section{ACKNOWLEDGEMENTS}

The Dutch organization for scientific research (NWO) is gratefully acknowledged for funding Eidetic (projectnumber: 634.000.001). In addition, Leon van den Broek and Janna von Schmid are acknowledged for resp. their help in calculation and for proof reading the manuscript.

\section{REFERENCES}

1. Berlin B. and Kay P. Basic color terms: Their universals and evolution. Berkeley: University of California Press, 1969.

2. Broek, E.L. van den, Hendriks M.A., Puts M.J.H., and Vuurpijl, L.G. Modeling human color categorization: Color discrimination and color memory. Proceedings of the BNAIC Conference 2003, University of Nijmegen Press, The Netherlands, 59-66.

3. Broek, E.L. van den, Kisters, P.M.F., \& Vuurpijl, L.G. The utilization of human color categorization for content-based image retrieval. In B.E. Rogowitz and T.N. Pappas (Eds.), Proceedings of Human Vision and Electronic Imaging $I X$, p. ?-?. January 18-22, U.S.A. San Jose, California. [in press]

4. Dix, A.J., Finlay, J.E., Abowd, G.D., and Beale R. Human-Computer Interaction. Pearson Education England, 1998.

5. Derefeldt, G., Swartling, T., Berggrund, U., and Bodrogi (2004). Cognitive color. Color, Research, \& Application, 29, 7-19.

6. Everly D. and Mason J.S. Color selection methods using the color picker. URL:

http://www.otal.umd.edu/SHORE99/jsmason/

7. Fitts, P.M. The information capacity of the human motor system in controlling the amplitude of movement. Journal of Experimental Psychology 47, 6 (1954), 381-391.

8. Lew, M. Next generation web searches for visual content. Computer 33, 11 (2000), 46-53.

9. Steiner, N. A review of web based color pickers. URL: http://www.web-graphics.com/feature-002.php

10. Venters, C.C. and Cooper, M. A review of contentbased image retrieval systems. http://www.jtap.ac.uk/reports/htm/jtap-054.html

11. Whorf, B.L. Language, thought, and reality: Selected papers of Benjamin Lee Whorf. Cambridge, M.A.: MIT Press, 1956. [Original work published 1941] 